\title{
Bell-Type Inequalities for Bivariate Maps on Orthomodular Lattices
}

\author{
Jarosław Pykacz ${ }^{1}$ • L’ubica Valášková ${ }^{2}$ • Ol'ga Nánásiová ${ }^{3}$
}

Received: 7 September 2014 / Accepted: 10 April 2015 / Published online: 19 May 2015

(C) The Author(s) 2015. This article is published with open access at Springerlink.com

\begin{abstract}
Bell-type inequalities on orthomodular lattices, in which conjunctions of propositions are not modeled by meets but by maps for simultaneous measurements ( $s$-maps), are studied. It is shown, that the most simple of these inequalities, that involves only two propositions, is always satisfied, contrary to what happens in the case of traditional version of this inequality in which conjunctions of propositions are modeled by meets. Equivalence of various Bell-type inequalities formulated with the aid of bivariate maps on orthomodular lattices is studied. Our investigations shed new light on the interpretation of various multivariate maps defined on orthomodular lattices already studied in the literature. The paper is concluded by showing the possibility of using $s$-maps and $j$-maps to represent counterfactual conjunctions and disjunctions of non-compatible propositions about quantum systems.
\end{abstract}

Keywords Bell-type inequalities $\cdot$ Orthomodular lattice $\cdot s$-map

\footnotetext{
\arosław Pykacz

pykacz@mat.ug.edu.pl

L’ubica Valášková

lubica.valaskova@stuba.sk

Ol'ga Nánásiová

olga.nanasiova@stuba.sk

1 Institute of Mathematics, University of Gdańsk, Gdańsk, Poland

2 Department of Mathematics and Descriptive Geometry, Faculty of Civil Engineering, Slovak University of Technology in Bratislava, Bratislava, Slovakia

3 Institute of Computer Science and Mathematics, Faculty of Electrical Engineering and Information Technology, Slovak University of Technology in Bratislava, Bratislava, Slovakia
} 


\section{Introduction}

Lindenbaum-Tarski algebras of theories in which propositions obey the rules of classical logic are Boolean algebras (BAs). In particular, meet $(\wedge)$ in these Boolean algebras describes conjunction of propositions. The same is usually assumed in the case of orthomodular lattices (OMLs) which are non-distributive generalizations of BAs, also in the case of lattices $\mathcal{L}(\mathcal{H})$ of projections onto closed linear subspaces of Hilbert spaces $\mathcal{H}$ used to describe quantum systems. Only rarely it is noticed that careless interpretation of meets in OMLs as always representing conjunction of propositions may lead to difficulties (see, e.g., [9]). Impossibility of simultaneous verification (noncompatibility) of some propositions about a physical system is a remarkable feature of quantum systems. However, in OMLs meet is a global operation, therefore it can be applied also to non-compatible elements of these lattices, which in the case of Hilbertian lattices $\mathcal{L}(\mathcal{H})$ makes its interpretation as a conjunction of propositions doubtful from the physical point of view.

Bell-type inequalities, studied intensively also in the realm of OMLs [3,5,6,22$25,30,31$ ] always contain terms interpreted as probabilities of conjunctions of propositions. In view of above-mentioned doubts concerning unrestricted treating of meets in OMLs as representing conjunctions of propositions, also interpretation of these inequalities becomes doubtful.

In order to get mathematical tools suitable for constructing virtual 'joint' probabilities of pairs of non-compatible propositions in OMLs, Nánásiová [12] introduced a notion of an $s$-map (map for simultaneous measurements) subsequently studied in numerous papers [1,13-17]. The aim of the present paper is to study Bell-type inequalities on OMLs in which probability of conjunction of propositions is modeled as a value that an $s$-map takes on a pair of these propositions instead of a probability of their meet.

\section{Bivariate Maps on Orthomodular Lattices}

We recall that an orthomodular lattice (OML) is a lattice $L$ with $0_{L}$ and $1_{L}$ as the smallest and the greatest element, respectively, endowed with a unary operation $a \mapsto$ $a^{\prime}$ such that the following conditions are satisfied:

(i) $a^{\prime \prime}:=\left(a^{\prime}\right)^{\prime}=a$;

(ii) $a \leq b$ implies $b^{\prime} \leq a^{\prime}$;

(iii) $a \vee a^{\prime}=1_{L}$;

(iv) $a \leq b$ implies $b=a \vee\left(a^{\prime} \wedge b\right)$.

Condition (iv) is called the orthomodular law. Elements of an OML are traditionally called propositions, although in the case when an OML is used as a basic structure of a generalized probability calculus, the name events is more appropriate. If an OML $L$ is closed under countable lattice operations, then $L$ is called a $\sigma$-orthomodular lattice $(\sigma$-OML). However, since in Bell-type inequalities only finite meets are concerned, in this paper we shall regard only the most basic case. In the quantum logic approach to quantum theory, $\sigma$-OMLs are considered as mathematical models of quantum mechanical propositions (see, e.g., [2,21]. In the traditional Hilbert space approach, this OML 
is a lattice $\mathcal{L}(\mathcal{H})$ of projections onto closed linear subspaces of the corresponding Hilbert space $\mathcal{H}$. More generally, the set of projections in every von Neumann algebra forms a complete OML [8].

Let $L$ be an OML. Two elements $a, b \in L$ are called orthogonal (denoted $a \perp b$ ) iff $a \leq b^{\prime}$, and $a, b$ are called compatible (denoted $\left.a \leftrightarrow b\right)$ iff $a=(a \wedge b) \vee\left(a \wedge b^{\prime}\right)$. Notice that two projections on a Hilbert space are orthogonal iff the product of them is zero, and compatible iff they commute.

A probability measure or state on $L$ is a mapping $m: L \rightarrow[0,1]$ such that

(i) $m\left(1_{L}\right)=1$;

(ii) $a \perp b$ implies $m(a \vee b)=m(a)+m(b)$.

A state $m$ is $\sigma$-additive if $m(a)=\sum_{i=1}^{\infty} m\left(a_{i}\right)$ whenever $a=\bigvee_{i=1}^{\infty} a_{i}$ for any sequence $\left\{a_{i}\right\}$ of pairwise orthogonal elements.

Let $L$ be an OML. A map $p: L \times L \rightarrow[0,1]$ is called a map for simultaneous measurements (abbr. s-map) [12] if the following conditions hold:

(s1) $p\left(1_{L}, 1_{L}\right)=1$;

(s2) if $a \perp b$, then $p(a, b)=0$;

(s3) if $a \perp b$, then for any $c \in L$ :

$$
\begin{aligned}
& p(a \vee b, c)=p(a, c)+p(b, c), \\
& p(c, a \vee b)=p(c, a)+p(c, b) .
\end{aligned}
$$

The following properties of $s$-maps proved in [12] will be of utmost importance in our considerations:

(N1) The map $m_{p}: L \rightarrow[0,1]$ such that $m_{p}(a)=p(a, a)=p\left(1_{L}, a\right)=p\left(a, 1_{L}\right)$ is a state on $L$. Such a state will be called a state generated by $p$.

(N2) If $a \leftrightarrow b$, then $p(a, b)=m_{p}(a \wedge b)=p(b, a)$. This property shows that $s$-maps can be seen as providing probabilities of 'virtual' conjunctions of propositions, even non-compatible ones, for in the case of compatible propositions $(a \leftrightarrow b)$ the value $p(a, b)$ coincides with the value that a state $m_{p}$ generated by $p$ takes on the meet $a \wedge b$, which in this case really represents conjunction of $a$ and $b$. (N3) $p\left(a^{\prime}, b^{\prime}\right)=1-p(a, a)-p(b, b)+p(a, b)$ for all elements of an OML.

Let us note that $s$-maps on OMLs resemble copulas (see, e.g., [19]) that are used in classical probability and statistics to construct joint probability distributions from the given marginal probability distributions.

It was shown many years ago by Greechie [7] that there exist OMLs admitting no states. Of course also no $s$-map can be defined on such OMLs, otherwise the abovementioned property of $s$-maps (N1) would not hold. On the other hand, there exist OMLs with abundance of $s$-maps, e.g., it was proved by Nánásiová and Pulmannová [15] that any tracial state on a von Neumann algebra with no type $I_{2}$ direct summand generates an $s$-map on the lattice of projections of this algebra. Also from Propositions 1.1 and 2.1 of Nánásiová [12] it follows that there exist a lot of $s$-maps on OMLs with unital sets of states.

In general the problem of existence of $s$-maps on various OMLs is far from being settled and deserves further investigations. However, since authors of numerous papers 
in which Bell-type inequalities on OMLs are formulated with the use of meets never bother about the existence of probability measures they use, we shall adopt the same position w.r.t. $s$-maps.

In ([18], see also $[16,17])$ the following notion of a join map $(j-$ map) on an OML has been introduced:

Let $L$ be an OML. A map $q: L \times L \rightarrow[0,1]$ is called a join map (abbr. $j$-map) if the following conditions hold:

(j1) $q\left(0_{L}, 0_{L}\right)=0, q\left(1_{L}, 1_{L}\right)=1$;

(j2) if $a \perp b$, then $q(a, b)=q(a, a)+q(b, b)$;

(j3) if $a \perp b$, then for any $c \in L$ :

$$
\begin{aligned}
& q(a \vee b, c)=q(a, c)+q(b, c)-q(c, c) \\
& q(c, a \vee b)=q(c, a)+q(c, b)-q(c, c) .
\end{aligned}
$$

It was proved in [16] that if $p$ is an $s$-map on an OML, then $q_{p}(a, b)=p(a, a)+$ $p(b, b)-p(a, b)=m_{p}(a)+m_{p}(b)-p(a, b)$ is a $j$-map, ${ }^{1}$ i.e., it maps $L \times L$ into $[0,1]$. This fact will be used as a straightforward justification of the most basic of Bell-type inequalities concerning $s$-maps on OMLs, that will be studied in the next section.

The third map $d: L \times L \rightarrow[0,1]$ which will be useful in our considerations, in Boolean case is a probability of symmetric difference $a \triangle b=\left(a \wedge b^{\prime}\right) \vee\left(a^{\prime} \wedge b\right)$ of two propositions. This is the reason for which it was called in $[16,17]$ (a difference map or simply a $d$-map. It is defined by the following conditions:

(d1) $d(a, a)=0$ for any $a \in L$, and $d\left(1_{L}, 0_{L}\right)=d\left(0_{L}, 1_{L}\right)=1$;

(d2) if $a \perp b$, then $d(a, b)=d\left(a, 0_{L}\right)+d\left(0_{L}, b\right)$;

(d3) if $a \perp b$, then for any $c \in L$ :

$$
\begin{aligned}
& d(a \vee b, c)=d(a, c)+d(b, c)-d\left(0_{L}, c\right) \\
& d(c, a \vee b)=d(c, a)+d(c, b)-d\left(c, 0_{L}\right)
\end{aligned}
$$

It was shown in [17] that as in the case of $j$-maps, each $s$-map $p$ on an OML induces a $d$-map $d_{p}$ by the formula: $d_{p}(a, b)=p\left(a, b^{\prime}\right)+p\left(a^{\prime}, b\right)$ (the reverse assertion is not true).

The following properties of the $d$-map $d_{p}$ induced by an $s$-map $p$ will be used in the sequel:

Lemma 1 Let $p$ be an s-map on an OML and $d_{p}$ be a d-map induced by $p$. Then

(a) $d_{p}(a, b)=p(a, a)+p(b, b)-2 p(a, b)$;

(b) $d_{p}\left(a, 0_{L}\right)=d_{p}\left(0_{L}, a\right)=p(a, a)=m_{p}(a)$.

\footnotetext{
$\overline{{ }^{1} \text { It is easy to see that if } a \leftrightarrow b \text {, then } q_{p}(a, b)=m_{p}(a)+m_{p}(b)}-m_{p}(a \wedge b)=m_{p}(a \vee b)$ which explains its name.
} 
Proof To show (a) it is enough to notice that from the condition (s3) of the definition of an $s$-map and its property (N1) it follows that $p(a, a)=p\left(a, 1_{L}\right)=p(a, b \vee$ $\left.b^{\prime}\right)=p(a, b)+p\left(a, b^{\prime}\right)$, so $p\left(a, b^{\prime}\right)=p(a, a)-p(a, b)$. Analogoulsly, $p\left(a^{\prime}, b\right)=$ $p(b, b)-p(a, b)$. By inserting these differences into the definition of $d_{p}$ we get (a).

Equalities (b) follow from (a) and from the fact that for any $a$ in an OML, $a \perp 0_{L}$, which by the condition (s2) of the definition of an $s$-map implies that $p\left(0_{L}, 0_{L}\right)=$ $p\left(a, 0_{L}\right)=0$.

\section{Bell-Type Inequalities in Which Probability of Conjunctions is Modeled by an $s$-Map}

We shall study $s$-map counterparts of the following Bell-type inequalities involving meets, that were studied already by Pitovsky [20] (see also [5,6,23]):

(B1) $m(a)+m(b)-m(a \wedge b) \leq 1$

(B2) $m(a)+m(b)+m(c)-m(a \wedge b)-m(a \wedge c)-m(b \wedge c) \leq 1$

(C1) $m(b)+m(c) \geq m(a \wedge b)+m(b \wedge c)+m(c \wedge d)-m(a \wedge d)$

(C2) $m(a \wedge b)+m(b \wedge c)+m(c \wedge d)-m(a \wedge d)-m(b)-m(c) \geq-1$.

Inequalities (B1) and (B2) are usually called inequalities of Bell-Wigner type while (C1) and (C2) are usually called inequalities of Clauser-Horne type. All these inequalities are satisfied by any probability measure on a BA.

Our aim is to study analogs of these inequalities in which, because of properties of $s$-maps mentioned in the previous section, probabilities of single events $m(a), m(b)$, etc. are replaced, respectively, by $p(a, a), p(b, b)$, etc., and probabilities of joint occurences of events are modeled by values that an $s$-map $p$ takes on pairs of events. This means that we shall study the following inequalities:

$\left(\mathrm{B} 1^{\prime}\right) p(a, a)+p(b, b)-p(a, b) \leq 1$

$\left(\mathrm{B} 2^{\prime}\right) p(a, a)+p(b, b)+p(c, c)-p(a, b)-p(a, c)-p(b, c) \leq 1$

$\left(\mathrm{C} 1^{\prime}\right) p(b, b)+p(c, c) \geq p(a, b)+p(b, c)+p(c, d)-p(a, d)$

$\left(\mathrm{C} 2^{\prime}\right) p(a, b)+p(b, c)+p(c, d)-p(a, d)-p(b, b)-p(c, c) \geq-1$.

Let us note, that since $s$-maps are in general non-commutative, each term $m(x \wedge y)$ in inequalities (B1)-(C2) yields two generalizations to $s$-maps: $p(x, y)$ and $p(y, x)$. Therefore, there are two $s$-map counterparts of inequality (B1), eight of (B2), and sixteen of $(\mathrm{C} 1)$ and $(\mathrm{C} 2)$, and inequalities $\left(\mathrm{B} 1^{\prime}\right)-\left(\mathrm{C} 2^{\prime}\right)$ form only a sample of them. However, we expect that this sample is a faithful representative of them all and we restrict our considerations to $\left(\mathrm{B} 1^{\prime}\right)-\left(\mathrm{C} 2^{\prime}\right)$. Moreover, it occurs that some particularly interesting problems concerning Bell-type inequalities for $s$-maps, that will be discussed in details in Sect. 4 appear only in the case of commuting $s$-maps, in which case inequalities $\left(\mathrm{B}^{\prime}\right)-\left(\mathrm{C} 2^{\prime}\right)$ are the unique $s$-map generalizations of inequalities (B1)-(C2).

It is straightforward to see that the expression on the left-hand side of inequality $\left(\mathrm{B} 1^{\prime}\right)$ is a $j$-map generated by an $s$-map $p$. Since any $j$-map takes values in the interval $[0,1]$, we obtain

Proposition 1 Inequality $\left(B 1^{\prime}\right)$ is satisfied by all s-maps defined on an $O M L$. 
This result is of utmost importance since it shows that $s$-maps, invented to describe probabilities of virtual 'joint' occurence of non-compatible events, are more close to classical probabilities than values $m(a \wedge b)$ usually interpreted as probabilities of coincidence of events $a$ and $b$. We stress once more that if events $a$ and $b$ are incompatible, the value $m(a \wedge b)$ cannot be checked in any experiment, so it can be replaced by any value that a bivariate map $p: L \times L \rightarrow[0,1]$ takes on a pair $a, b \in L$, provided that $p(a, b)=m(a \wedge b)$ if $a \leftrightarrow b$. This requirement is met by $s$-maps. Actually, $s$-maps were invented just to meet this requirement! In view of this result, the fact that inequality (B1) may be violated by probability measures defined on an OML is for quantum physics of no importance at all, since it shows that this violation can occur only for non-compatible propositions, i.e., it can be never checked experimentally.

\subsection{Bell-Wigner Inequality $\left(\mathrm{B2}^{\prime}\right)$ for $s$-Map is a Triangle Inequality for Induced $d$-Map}

In the case of compatible propositions ( $a \leftrightarrow b$ ) the expression in the part (a) of Lemma 1 takes the form: $d_{p}(a, b)=m_{p}(a)+m_{p}(b)-2 m_{p}(a \wedge b)$, therefore, if $a \leftrightarrow b$, $d_{p}(a, b)$ coincides with the notion of separation of $a$ and $b$ defined in this way by Santos already in 1986. Santos in his paper [31] proved that if an OML is a Boolean algebra, then separation fulfills triangle inequality, in fact it is a pseudometric on an OML. ${ }^{2}$ Moreover, he proved that triangle inequality for a separation is equivalent to the Belltype inequality (C1). Since our $d$-map $d_{p}(a, b)$ coincides with Santos'separation for a state $m_{p}$ on all pairs of compatible propositions, it is interesting to study connections of Bell-type inequalities for an $s$-map $p(a, b)$ with triangle inequalities for the induced $d$-map $d_{p}(a, b)$. In this respect we get the following:

Lemma 2 Let $L$ be an $O M L$, let $p$ be an s-map on $L$, and $d_{p}$ be a d-map induced by p. Bell-Wigner inequality $\left(B 2^{\prime}\right)$ for the s-map p is equivalent to the following triangle inequality for the $d$-map $d_{p}$ :

$(\triangle) d_{p}(a, c) \leq d_{p}\left(a, b^{\prime}\right)+d_{p}\left(b^{\prime}, c\right)$.

Moreover, the Clauser-Horne type inequalities $\left(C 1^{\prime}\right)$ and $\left(C 2^{\prime}\right)$ are equivalent, respectively, to the following inequalities:

$\left(\triangle^{\prime}\right) d_{p}(a, d) \leq d_{p}(a, b)+d_{p}(b, c)+d_{p}(c, d)$

$\left(\triangle^{\prime \prime}\right) d_{p}(a, b)+d_{p}(b, c)+d_{p}(c, d) \leq 2+d_{p}(a, d)$.

Proof The proof of equivalence $\left(\mathrm{B} 2^{\prime}\right) \Leftrightarrow(\triangle)$ goes through the following sequence of equivalent inequalities, where the differences of the type $p\left(a, b^{\prime}\right)=p(a, a)-p(a, b)$, already used in the proof of Lemma 1 , and also the fact that $p\left(b^{\prime}, b^{\prime}\right)=m_{p}\left(b^{\prime}\right)=$ $1-m_{p}(b)=1-p(b, b)$ are applied:

\footnotetext{
${ }^{2}$ A mapping $d: L \times L \rightarrow[0,1]$ is a pseudometric on $L$ if for all $a, b, c \in L: d(a, a)=0 ; d(a, b)=$ $d(b, a) ; d(a, b) \leq d(a, c)+d(c, b)$.
} 


$$
\begin{aligned}
d_{p}(a, c) \leq & d_{p}\left(a, b^{\prime}\right)+d_{p}\left(b^{\prime}, c\right) \\
p(a, a)+p(c, c)-2 p(a, c) \leq & \left(p(a, a)+p\left(b^{\prime}, b^{\prime}\right)-2 p\left(a, b^{\prime}\right)\right) \\
& +\left(p\left(b^{\prime}, b^{\prime}\right)+p(c, c)-2 p\left(b^{\prime}, c\right)\right) .
\end{aligned}
$$

It means that

$$
\begin{gathered}
-2 p(a, c) \leq 2 p\left(b^{\prime}, b^{\prime}\right)-2 p\left(a, b^{\prime}\right)-2 p\left(b^{\prime}, c\right) \\
p\left(a, b^{\prime}\right)+p\left(b^{\prime}, c\right)-p(a, c)-p\left(b^{\prime}, b^{\prime}\right) \leq 0 \\
(p(a, a)-p(a, b))+(p(c, c)-p(b, c))-p(a, c)-(1-p(b, b)) \leq 0 \\
p(a, a)+p(b, b)+p(c, c)-p(a, b)-p(b, c)-p(a, c) \leq 1 .
\end{gathered}
$$

The proof of equivalence $\left(\mathrm{C1}^{\prime}\right) \Leftrightarrow\left(\triangle^{\prime}\right)$ also goes through the sequence of equivalent inequalities. Let us begin with $\left(\triangle^{\prime}\right)$ :

$$
d_{p}(a, d) \leq d_{p}(a, b)+d_{p}(b, c)+d_{p}(c, d) .
$$

By condition (a) of Lemma 1 this inequality is equivalent to the following inequality:

$$
-2 p(a, d) \leq 2 p(b, b)-2 p(a, b)+2 p(c, c)-2 p(b, c)-2 p(c, d) .
$$

It means that

$$
2 p(a, b)+2 p(b, c)+2 p(c, d)-2 p(a, d) \leq 2 p(b, b)+2 p(c, c),
$$

so

$$
p(a, b)+p(b, c)+p(c, d)-p(a, d) \leq p(b, b)+p(c, c)
$$

The proof of equivalence $\left(\mathrm{C} 2^{\prime}\right) \Leftrightarrow\left(\triangle^{\prime \prime}\right)$ is analogous.

Remark 1 Let us note that equivalences $\left(\mathrm{C}^{\prime}\right) \Leftrightarrow\left(\triangle^{\prime}\right)$ and $\left(\mathrm{C}^{\prime}\right) \Leftrightarrow\left(\triangle^{\prime \prime}\right)$ are 'faithful' ones, in the sense that the same elements appear on both sides of these equivalences. This does not happen in the case of equivalence $\left(B 2^{\prime}\right) \Leftrightarrow(\triangle)$, but of course the following corollary holds:

Corollary 1 If one of the inequalities $\left(B 2^{\prime}\right)$ or $(\triangle)$ is satisfied by all triples of elements of an $O M L$, the same happens for the other one. 


\subsection{Equivalence of Bell-Type Inequalities Involving More Than Two Elements}

According to Proposition 1 the simplest Bell-type inequality (B1') that involves only two elements is always satisfied by any $s$-map defined on an OML. The following example shows that this does not have to happen in the case of Bell-type inequalities that involve more than two elements.

Example 1 Let $L$ be a horizontal sum of three Boolean algebras $2^{2}$ :

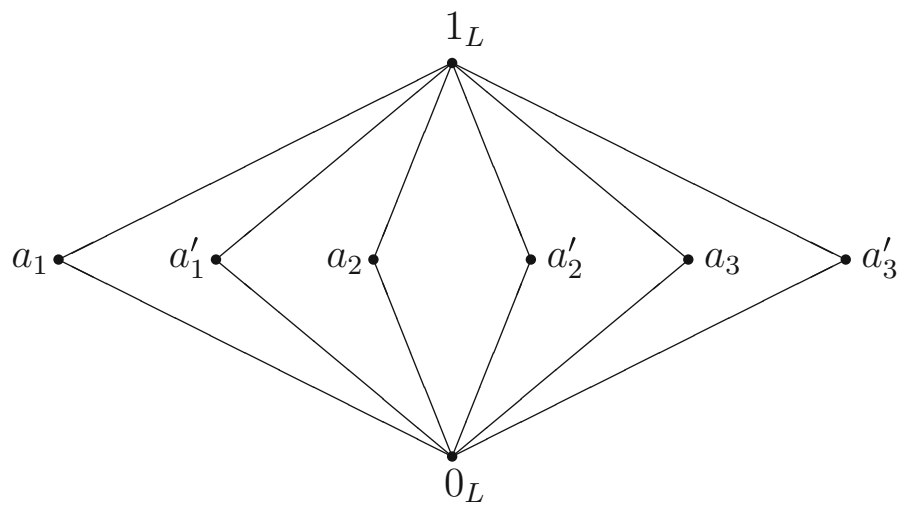

Let $p$ be a commutative bivariate map defined on $L$ in the following way $\forall i, j=$ $1,2,3 ; i \neq j$ :

$$
\begin{aligned}
p\left(1_{L}, 1_{L}\right) & =1, \quad p\left(0_{L}, 0_{L}\right)=0, \quad p\left(1_{L}, 0_{L}\right)=0 ; \\
p\left(0_{L}, a_{i}\right) & =p\left(0_{L}, a_{i}^{\prime}\right)=p\left(a_{i}, a_{i}^{\prime}\right)=0 ; \\
p\left(a_{i}, a_{i}\right) & =p\left(a_{i}^{\prime}, a_{i}^{\prime}\right)=p\left(a_{i}, 1_{L}\right)=p\left(a_{i}^{\prime}, 1_{L}\right)=0.5 ; \\
p\left(a_{i}, a_{j}\right) & =0.1 ; \\
p\left(a_{i}, a_{j}^{\prime}\right) & =p\left(a_{i}, a_{i}\right)-p\left(a_{i}, a_{j}\right)=0.4 ; \\
p\left(a_{i}^{\prime}, a_{j}^{\prime}\right) & =1-p\left(a_{i}, a_{i}\right)-p\left(a_{j}, a_{j}\right)+p\left(a_{i}, a_{j}\right)=0.1 .
\end{aligned}
$$

It is easy to check that $p$ is an $s$-map which, however, does not satisfy Bell-Wigner inequality $\left(\mathrm{B} 2^{\prime}\right)$ :

$$
p\left(a_{1}, a_{1}\right)+p\left(a_{2}, a_{2}\right)+p\left(a_{3}, a_{3}\right)-p\left(a_{1}, a_{2}\right)-p\left(a_{2}, a_{3}\right)-p\left(a_{1}, a_{3}\right) \leq 1
$$

because

$$
3 \cdot 0.5-3 \cdot 0.1>1
$$

However, due to results obtained in the previous subsection, we can prove the following:

Proposition 2 Bell-Wigner inequalities $\left(B 2^{\prime}\right)$ and triangle inequalities $(\triangle)$ are satisfied for all triples of elements of an OML if and only if inequalities $\left(\triangle^{\prime}\right)$ and $\left(C 1^{\prime}\right)$ are 
satisfied for all quadruples of elements of an OML. Moreover, if an s-map involved in these inequalities is commutative, also inequalities $\left(\triangle^{\prime \prime}\right)$ and $\left(C 2^{\prime}\right)$ are satisfied.

Proof According to Corollary 1 in this case we can write triangle inequality $(\triangle)$ in the usual form: $d_{p}(a, c) \leq d_{p}(a, b)+d_{p}(b, c)$. Then its equivalence with quadrilateral inequality $\left(\triangle^{\prime}\right)$ can be shown in a standard way: $(\triangle) \Rightarrow\left(\triangle^{\prime}\right)$ because

$$
d_{p}(a, d) \leq d_{p}(a, b)+d_{p}(b, d) \leq d_{p}(a, b)+d_{p}(b, c)+d_{p}(c, d),
$$

and the opposite implication is obtained by substitution $d \mapsto c$ in the quadrilateral inequality $\left(\triangle^{\prime}\right)$.

Since $\left(\mathrm{C}^{\prime}\right) \Leftrightarrow\left(\triangle^{\prime}\right)$ and $\left(\mathrm{C} 2^{\prime}\right) \Leftrightarrow\left(\triangle^{\prime \prime}\right)$, to finish the proof it suffices to show that $\left(\mathrm{C}^{\prime}\right) \Leftrightarrow\left(\mathrm{C} 2^{\prime}\right)$ or $\left(\triangle^{\prime}\right) \Leftrightarrow\left(\triangle^{\prime \prime}\right)$. We shall show the first equivalence substituting $a \mapsto c$, $b \mapsto b^{\prime}$, and $c \mapsto a$ in $\left(\mathrm{Cl}^{\prime}\right)$. Then, using the same substitutions as in previous proofs, we obtain a sequence of equivalent inequalities, where, however, commutativity of the $s$-map that appears in these inequalities, is utilized:

$$
\begin{gathered}
p\left(b^{\prime}, b^{\prime}\right)+p(a, a) \geq p\left(c, b^{\prime}\right)+p\left(b^{\prime}, a\right)+p(a, d)-p(c, d) \\
1-p(b, b)+p(a, a)-(p(c, c)-p(c, b))-(p(a, a)-p(a, b))-p(a, d)+p(c, d) \geq 0 \\
-p(b, b)+p(a, a)-p(c, c)+p(c, b)-p(a, a)+p(a, b)-p(a, d)+p(c, d) \geq-1 \\
-p(b, b)-p(c, c)+p(a, b)+p(b, c)+p(c, d)-p(a, d) \geq-1 .
\end{gathered}
$$

\section{Bell-Type Inequalities and Existence of Multivariate $s$-Maps}

In [14] the notion of an $s$-map was generalized to the notion of $n$-variate $s$-map (abbr. $s_{n}$-map) in the following way:

Let $L$ be an OML. A map $p_{n}: L^{n} \rightarrow[0,1]$ is called an $s_{n}$-map if the following conditions hold:

$\left(\mathrm{s}_{n} 1\right) p_{n}\left(1_{L}, \ldots, 1_{L}\right)=1$;

$\left(\mathrm{s}_{n} 2\right)$ if $a_{i} \perp a_{j}$ for some $i \neq j$, then $p_{n}\left(a_{1}, \ldots, a_{n}\right)=0$;

$\left(\mathrm{s}_{n} 3\right)$ if $a_{i} \perp b_{i}$ for some $i$, then for all $c_{1}, \ldots, c_{i-1}, c_{i+1}, \ldots, c_{n} \in L$

$$
p_{n}\left(c_{1}, \ldots, a_{i} \vee b_{i}, \ldots, c_{n}\right)=p_{n}\left(c_{1}, \ldots, a_{i}, \ldots, c_{n}\right)+p_{n}\left(c_{1}, \ldots, b_{i}, \ldots, c_{n}\right) .
$$

The aim of introducing $s_{n}$-maps was to construct joint probability distributions of more than two non-compatible observables. The properties of multivariate $s_{n}$-maps are the same as properties of bivariate $s$-maps (which, actually, are $s_{2}$-maps).

Loosely speaking, in classical probability theory from any joint probability distribution one can obtain marginal probability distributions by replacing some random 
events by the sure event $\Omega$. Inspired by this, Nánásiová and Khrennikov [14] defined, for a given $s_{n}$-map $p_{n}$ and every $k<n$, a marginal $s_{k}$-map by the formula:

$$
p_{k}: L^{k} \rightarrow[0,1], \quad p_{k}\left(a_{1}, a_{2}, \ldots, a_{k}\right)=p(\underbrace{a_{1}, a_{2}, \ldots, a_{k}, 1_{L}, \ldots, 1_{L}}_{n})
$$

Although in general $s_{n}$-maps are not invariant with respect to permutations, they proved that this happens when there exist two arguments of an $s_{n}$-map that are compatible. Therefore, in the definition of a marginal $s_{k}$-map the maximal element $1_{L}$ of an OML can be placed at any position. They also proved that an $s_{k}$-map that is a marginal map of some $s_{n}$-map with $n>k$ is always invariant with respect to permutations. In particular, this means that in the case of bivariate $s$-maps, all of them that are marginal $s_{2}$-maps of some $s_{3}$-maps, are commutative.

In numerous papers written by members of the so called 'probabilistic opposition' 3 to the usual interpretation of violation of Bell-type inequalities (see [10] and references cited therein), violation of Bell-type inequalities is not ascribed to nonlocality or lack of realism, but rather results from non-existence of a joint probability distribution that could yield marginal distributions being in accordance with probabilities obtained from quantum-mechanical calculations. In our 'meet-free' approach we get the following result:

Proposition 3 Let $L$ be an OML and let p be a bivariate commutative $s_{2}$-map on L. If for some $a, b, c \in L$ Bell-Wigner inequality $\left(B 2^{\prime}\right)$ is not satisfied, then a trivariate $s_{3}$ map with marginal bivariate $s_{2}$-map p does not exist. On the other hand, if a trivariate $s_{3}$-map $p^{\prime}$ exists, then Bell-Wigner inequality $\left(B 2^{\prime}\right)$ is satisfied by all marginal $s_{2}$-maps obtained from $p^{\prime}$.

Proof If Bell-Wigner inequality (B2') is not satisfied for some $a, b, c \in L$, then $1-p(a, a)-p(b, b)-p(c, c)+p(a, b)+p(a, c)+p(b, c)<0$. Let us assume that trivariate $s$-map $p_{3}$ with marginal bivariate $s$-map $p$ exists. Then $p_{3}\left(x, y, 1_{L}\right)=$ $p_{3}\left(x, 1_{L}, y\right)=p_{3}\left(1_{L}, x, y\right)=p(x, y)$. Let us denote $p_{3}(a, b, c)=\alpha \in[0,1]$. Using definition of marginal $s_{2}$-maps and property (N3) of $s$-maps we get:

$$
\begin{aligned}
p_{3}\left(a^{\prime}, b^{\prime}, c^{\prime}\right) & =p_{3}\left(a^{\prime}, b^{\prime}, 1_{L}\right)-p_{3}\left(a^{\prime}, b^{\prime}, c\right) \\
& =p\left(a^{\prime}, b^{\prime}\right)-\left(p_{3}\left(a^{\prime}, 1_{L}, c\right)-p_{3}\left(a^{\prime}, b, c\right)\right) \\
& =p\left(a^{\prime}, b^{\prime}\right)-p\left(a^{\prime}, c\right)+\left(p_{3}\left(1_{L}, b, c\right)-p_{3}(a, b, c)\right) \\
& =p\left(a^{\prime}, b^{\prime}\right)-p\left(a^{\prime}, c\right)+p(b, c)-\alpha \\
& =(1-p(a, a)-p(b, b)+p(a, b))-(p(c, c)-p(a, c))+p(b, c)-\alpha \\
& =1-p(a, a)-p(b, b)-p(c, c)+p(a, b)+p(a, c)+p(b, c)-\alpha .
\end{aligned}
$$

Therefore, the value of $p_{3}\left(a^{\prime}, b^{\prime}, c^{\prime}\right)$ is negative for any $\alpha \in[0,1]$, which is impossible.

The second part of Proposition follows directly from its first part.

3 A term coined by Khrennikov and used by him, e.g., in [10]. 
Proposition 3 shows that even within the, we dare say, more correct approach, in which probabilities of conjunctions of propositions are not calculated as values that probability measures defined on OMLs take on meets of elements of an OML, but rather as values that $s$-maps take on pairs of these propositions, violation of BellWigner inequality (B2') means that a (generalized) joint probability distribution that could be used to describe the experimental situation does not exist.

\section{The Logic of Counterfactual Propositions About Quantum Systems}

As it was mentioned in the Introduction, in Boolean algebras which are algebraic representation of families of experimentally verifiable propositions pertaining to classical physical systems, meets and joins are proper models of conjunctions and disjunctions of propositions. However, unrestricted generalization of this statement to OMLs that are algebraic representations of 'quantum logics', i.e., sets of experimentally verifiable propositions pertaining to quantum systems, leads to numerous difficulties caused by the fact that it is not possible to verify simultaneously propositions which are represented by non-compatible elements of an OML. (In order to simplify the language such propositions about quantum systems will be themselves called non-compatible in the sequel. We shall also often identify propositions about quantum system with elements of an OML that represent them). Actually, according to the strict 'verificationist' point of view, conjunctions and disjunctions of non-compatible propositions should be regarded as meaningless.

According to the traditional approach, originated by Birkhoff and von Neumann in their historic paper [4] 'quantum logic' is regarded as 2-valued logic, which is nonclassical because of non-distributivity. However, one of the authors in a series of papers (see, e.g., [26-28]) promoted an idea that 'quantum logic' can be equivalently regarded as a specific $\infty$-valued Łukasiewicz logic, which opens the possibility of working out a new interpretation of quantum mechanics [29]. In this approach conjunctions and disjunctions of propositions about quantum systems are modelled by a pair of partially defined operations used in a specific version of Łukasiewicz many-valued logic. However, when they are defined, they necessarily coincide with meets and joins [27]. Therefore, similarly to lattice operations of meet and join, they cannot be treated as proper models of conjunctions and disjunctions of non-compatible propositions.

The notion of an $s$-map opens a new possibility: if propositions $a$ and $b$ are non-compatible, the value $p(a, b)$ can be thought of as representing probability of simultaneous verification of $a$ and $b$ in a 'counterfactual measurement': 'what would be the probability of simultaneous verification of propositions a and $b$ if we were able to perform it' or, according to the approach propounded in [26-28] 'what would be the truth-value of the "counterfactual conjunction" of propositions a and b'. Let us remind that the traditional belief that $m(a \wedge b)$ always represents probability of simultaneous verification of propositions $a$ and $b$ is based on the tacit, and in the case of non-compatible propositions erroneous assumption, that this simultaneous verification is always possible.

Similarly, the value $q_{p}(a, b)=p(a, a)+p(b, a)-,p(a, b)$ can be thought of as representing truth-value of 'counterfactual disjunction' of propositions represented by $a$ and $b$. 
Therefore, we see that an $s$-map $p: L \times L \rightarrow[0,1]$ and its associated $j$-map $q_{p}$ allow to define for a studied quantum system a kind of a 'logic of counterfactuals' in which truth-values of a counterfactual conjunction $a \triangle_{p} b$ and a counterfactual disjunction $a \nabla_{p} b$ of two non-compatible propositions are modelled by $p(a, b)$ and $q_{p}(a, b)$, respectively.

Let us note that

$$
p\left(a^{\prime}, b^{\prime}\right)=1-p(a, a)-p p(a, b)+p(a, b)=1-q_{p}(a, b)
$$

and

$$
\begin{aligned}
q_{p}\left(a^{\prime}, b^{\prime}\right) & =p\left(a^{\prime}, a^{\prime}\right)+p\left(b^{\prime}, b^{\prime}\right)-p\left(a^{\prime}, b^{\prime}\right) \\
& =1-p(a, a)+1-p(b, b)-1+p(a, a)+p(b, b)-p(a, b) \\
& =1-p(a, b) .
\end{aligned}
$$

If we assume, as usual, that orthocomplementation in an OML represents logical negation and, as it is always assumed in Łukasiewicz many-valued logic [11], that truth-values of a proposition and its negation sum up to 1, we recognize in the formulas written above numerical expressions of both De Morgan laws:

$$
\begin{aligned}
& a^{\prime} \triangle_{p} b^{\prime}=\left[a \nabla_{p} b\right]^{\prime}, \\
& a^{\prime} \nabla_{p} b^{\prime}=\left[a \triangle_{p} b\right]^{\prime} .
\end{aligned}
$$

Since conjunction and disjunction of compatible propositions are properly modelled by their meet and join, the validity of numerous other laws, like the law of excluded middle, the law of contradiction and the orthomodular law, is secured by properties of meet and join in OMLs. Therefore, using $s$-maps and associated with them $j$-maps, we have obtained a kind of an 'extended quantum logic' in which conjunction and disjunction is meaningful both in the case of compatible, as well as non-compatible propositions.

\section{Summary}

Since interpretation of meets as representing conjunctions of propositions about quantum-mechanical systems is doubtful when these propositions are non-compatible, we studied various Bell-type inequalities on OMLs in which probabilities of meets of propositions were replaced by values that an $s$-map-an object invented to model probabilities of simultaneous measurements of incompatible propositions-takes on these propositions. It is significant that although the simplest Bell-type inequality (B1): $m(a)+m(b)-m(a \wedge b) \leq 1$ may be violated on an OML (of course only by noncompatible elements), its $s$-map counterpart (B1'): $p(a, a)+p(b, b)-p(a, b) \leq 1$ is always satisfied. This shows that replacing $m(a \wedge b)$ by $p(a, b)$ brings us closer to the situation encountered in classical probability theory, hopefully also closer to reality. 
Nevertheless, Proposition 3 shows that even within our approach violation of BellWigner inequality $\left(\mathrm{B} 2^{\prime}\right)$ by an $s_{2}$-map $p$ means that there does not exist an $s_{3}$-map for which $p$ would be a marginal $s_{2}$-map. This is in accordance with numerous papers written by 'probabilistic opposition' to the usual interpretation of violation of Belltype inequalities, in which violation of Bell-type inequalities is not ascribed to nonexistence of 'local realism', but rather indicates impossibility of constructing a single probability space in which experiments designed to check Bell-type inequalities could be embedded. Whether this impossibility follows from, or is equivalent to, the nonexistence of 'local realism', should be the aim of further investigations.

Finally, we showed that one can treat values that an $s$-map $p$ takes on noncompatible propositions about quantum systems as truth-values of 'counterfactual conjunctions' of these propositions, and similarly values that an associated $j$-map $q_{p}$ takes on such propositions as truth-values of 'counterfactual disjunctions' of them. This allows to construct propositional calculus ('extended quantum logic') in which conjunctions and disjunctions of both compatible and non-compatible propositions are meaningful.

Acknowledgments JP gratefully acknowledges financial support of the Polish National Center for Science (NCN) under the Grant 2011/03/B/HS1/04573 and a scholarship of the National Scholarship Programme of the Slovak Republic which enabled him to stay three months in Bratislava and work on this paper. ON and LV acknowledge financial support by the Grants VEGA 1/0143/11 and VEGA 1/0297/11.

Open Access This article is distributed under the terms of the Creative Commons Attribution 4.0 International License (http://creativecommons.org/licenses/by/4.0/), which permits unrestricted use, distribution, and reproduction in any medium, provided you give appropriate credit to the original author(s) and the source, provide a link to the Creative Commons license, and indicate if changes were made.

\section{References}

1. Al-Adilee, A., Nánásiová, O.: Copula and s-map on a quantum logic. Inf. Sci. 179, 4199-4207 (2009)

2. Beltrametti, E., Cassinelli, G.: The Logic of Quantum Mechanics. Addison-Wesley, Reading (1981)

3. Beltrametti, E., Mączyński, M.: Problem of classical and nonclassical probabilities. Int. J. Theor. Phys. 31, 1849-1856 (1992)

4. Birkhoff, G., von Neumann, J.: The logic of quantum mechanics. Ann. Math. 37, 823-843 (1936)

5. Dvurečenskij, A., Länger, H.: Bell-type inequalities in orthomodular lattices, I. Int. J. Theor. Phys. 34, 995-1024 (1995)

6. Dvurečenskij, A., Länger, H.: Bell-type inequalities in orthomodular lattices, II. Int. J. Theor. Phys. 34, 1025-1036 (1995)

7. Greechie, R.: Orthomodular lattices admitting no states. J. Comb. Theory 10, 119-132 (1971)

8. Hamhalter, J.: Quantum Measure Theory. Kluwer, Dordrecht (2003)

9. Jammer, M.: The Philosophy of Quantum Mechanics. Wiley-Interscience, New York (1974)

10. Khrennikov, A.: Violation of Bell's inequality and non-Kolmogorovness. In: Accardi, L., et al. (eds.) Foundations of Probability and Physics-5. American Institute of Physics, Mellville (2009)

11. Łukasiewicz, J.: Selected Works. North Holland, Amsterdam (1970)

12. Nánásiová, O.: Map for simultaneous measurements for a quantum logic. Int. J. Theor. Phys. 42, 1889-1903 (2003)

13. Nánásiová, O., Khrennikov, A.: Representation theorem for observables on a quantum system. Int. J. Theor. Phys. 45, 481-494 (2006)

14. Nánásiová, O., Khrennikov, A.: Compatibility and marginality. Int. J. Theor. Phys. 46, 1083-1095 (2007)

15. Nánásiová, O., Pulmannová, S.: S-map and tracial states. Inf. Sci. 179, 515-520 (2009)

16. Nánásiová, O., Valášková, L.: Maps on a quantum logic. Soft Comput. 14, 1047-1052 (2010) 
17. Nánásiová, O., Valášková, L.: Marginality and triangle inequality. Int. J. Theor. Phys. 49, 3199-3208 (2010)

18. Nánásiová, O., Minárová, M., Mohammed, A.: Probability and quantum logic. Forum Stat. Slovacum 5(2007), 101-107 (2007)

19. Nielsen, R.B.: An Introduction to Copulas. Springer, New York (1999)

20. Pitovsky, I.: Quantum Probability-Quantum Logic. Springer, Berlin (1989)

21. Pták, P., Pulmannová, S.: Orthomodular Structures as Quantum Logics. Kluwer, Dordrecht (1991)

22. Pulmannová, S.: Bell inequalities and quantum logics. In: Accardi, L. (ed.) The Interpretations of Quantum Theory: Where Do We Stand?. Encyclopedia Italiana, Roma (1994)

23. Pulmannová, S.: Hidden variables and Bell inequalities on quantum logics. Found. Phys. 32, 193-216 (2002)

24. Pulmannová, S., Majernik, V.: Bell inequalities on quantum logics. J. Math. Phys. 33, 2173-2178 (1992)

25. Pykacz, J.: On Bell type inequalities in quantum logics. In: Bitsakis, E.J., Nicolaides, C.A. (eds.) Concept Probability. Kluwer, Dordrecht (1989)

26. Pykacz, J.: Fuzzy quantum logic and infinite-valued Łukasiewicz logic. Int. J. Theor. Phys. 33, 14031416 (1994)

27. Pykacz, J.: Łukasiewicz operations in fuzzy set and many-valued representations of quantum logics. Found. Phys. 30, 1503-1524 (2000)

28. Pykacz, J.: Unification of two approaches to quantum logic: every Birkhoff-von Neumann quantum logic is a partial infinite-valued Łukasiewicz logic. Stud. Log. 95, 5-20 (2010)

29. Pykacz, J.: Towards many-valued/fuzzy interpretation of quantum mechanics. Int. J. Gen. Syst. 40, $11-21(2011)$

30. Pykacz, J., Santos, E.: Hidden variables in quantum logic approach revisited. J. Math. Phys. 32, 12871292 (1991)

31. Santos, E.: The Bell inequalities as tests of classical logic. Phys. Lett. A 115, 363-365 (1986) 